\title{
The Application of Double-layer Curtains for Shaping Acoustics of Concert Halls
}

\author{
A. Pilch*, A. Karlińska, A. Snakowska And T. Kamisiński \\ AGH University of Science and Technology, Faculty of Mechanical Engineering and Robotics \\ Department of Mechanics and Vibroacoustics \\ Al. A. Mickiewicza 30, 30-059 Krakow, Poland
}

\begin{abstract}
Requirements concerning adjustment of acoustical parameters are nowadays more and more frequently numbered among design assumptions adopted for newly constructed concert halls and other auditoriums planned to play different functions. Investors usually require that the newly designed interiors can be used for such different purposes as playing orchestral music, performing theatre spectacles, screening movies, or holding lectures. This means the necessity to undertake studies on new technologies allowing to excert effective control over acoustics of multipurpose auditoriums. This paper proposes to use acoustic curtains as an effective means of modification of acoustic absorptivity of rooms. A design solutions for such curtains is described together with basics of the theory allowing to predict their acoustic properties. Results of sound absorption coefficient calculations performed for selected systems on the grounds of airflow resistivity values assumed for the used fabrics are verified by means of results of measurements performed in the impedance tube. It is proven that double-layer curtains show more balanced sound absorption characteristics compared to single-layer curtains and therefore represent a more convenient option when used for acoustical adaptation of rooms. Based on model studies, design solutions with double-layer curtains is developed for two concert halls that allow to adjust acoustic properties of the interiors to the required functions.
\end{abstract}

DOI: 10.12693/APhysPolA.125.A-113

PACS: 43.55.-n, 43.55.Dt, 43.58.-e

\section{Introduction}

The parameter used most universally for evaluation of interiors with classified acoustics is the reverberation time. The quantity is closely related with such fundamental parameters characterizing the acoustic quality of auditoriums as the speech intelligibility and the sound clarity factor C80 (according to Beranek [1], the correlation coefficient is -0.84). Advantages of using the reverberation time for valuation of auditorium halls include simplicity of the measurement and predictability of results obtained in the design work based on knowledge of sound absorption coefficient values for materials distributed within a defined interior volume [2]. These parameters are usually subjected to modification in order to control acoustics of the room in question. It is also possible to manipulate diffuseness of the sound field as a method of varying room's acoustics [3] but it is very difficult to measure that parameter, so it is used only as a support for an absorption change.

The easiest method to influence acoustics of a room consists in changing the surface area of a construction material characterized with high absorption coefficient value. This approach is frequently embodied by installing textile acoustic curtains. Merits of structures of that type include possibility to shape their acoustic parameters in a wide range by changing the density and airflow resistance of materials used to construct them [4] and easi-

*corresponding author; e-mail: apilch@agh.edu.pl ness to give the curtains different spatial forms, mainly by draping them and adjusting distance to walls and between individual fabric layers in multilayer structures [5]. Modification of these parameters allows to adjust the reverberation time of the room subjected to adaptation in accordance with demands specific for different functional programs, including those involving artificial sound amplification where lower reverberation times are more desirable.

In the framework of the present study, the concert hall of the Podkarpacka Philharmonic has been analyzed as an interior utilized frequently for theatre performances with the use of the sound amplification system. It has been proposed that the rear stage wall would be provided with a double-layer curtain designed in such a way that a flat reverberation time characteristic of the room is obtained.

A further application of curtains consists in using them for maintaining constant acoustic conditions in concert halls regardless of the degree of occupation. The Krakow Philharmonic concert hall was subjected to tests and analyses resulting in determination of actual possibility to maintain a constant reverberation time characteristics on the stage for varying degree of occupation thanks to the use of double-layer curtains.

\section{Analytical modeling of the sound absorption} coefficient for fabric structures

Absorption of sound by acoustic textile structures is influenced mainly by airflow resistivity of the used materials that for a porous fabric with thickness $d$ is defined as ratio of the pressure difference $\Delta P$ on both sides of the material to the product of the linear air flow rate $u$ through the material and its thickness [6]: 


$$
\sigma=\frac{\Delta P}{u d} .
$$

The airflow resistivity affects mainly the real part of acoustic impedance defined as the ratio of the acoustic pressure $p$ on surface of the material and the normal component of the acoustic velocity $v$ at the material surface that is generated by this very acoustic pressure characterizing the incident wave [7]:

$$
Z=\left(\frac{p}{v_{n}}\right)_{\text {surface }} .
$$

Imaginary part of the acoustic impedance is determined mainly by the structural impedance $z_{s}$ that for perfectly susceptible materials can be expressed by means of the relationship [7]

$$
z_{s}=-\mathrm{i} \omega m
$$

where $\omega=2 \pi f$ is the angular frequency, $f$ is the frequency, and $m$ is the surface mass density of the analyzed material.

The specific acoustic impedance of a textile material can be expressed as a parallel combination of the normalized acoustic impedance $z$ related to flow of air through the fabric and the normalized structural acoustic impedance of the material $z_{s}[8]$ :

$$
z_{e}=\frac{z z_{s}}{z+z_{s}} \text {. }
$$

The above relationship, by introducing real and imaginary parts of the acoustic impedance $z$ according to definition

$$
z=r+\mathrm{i} x,
$$

where $r=\sigma d$, can be expressed in the form [8]

$$
z_{e}=\frac{r(\omega m)^{2}}{r^{2}+(\omega m-x)^{2}}+i \omega m \frac{r^{2}-x(\omega m-x)}{r^{2}+(\omega m-x)^{2}} .
$$

Textile surfaces are characterized with negligible acoustic reactance, so $x=0$ will be assumed in what follows [9]. Continuing calculations of acoustic impedance of the sys-

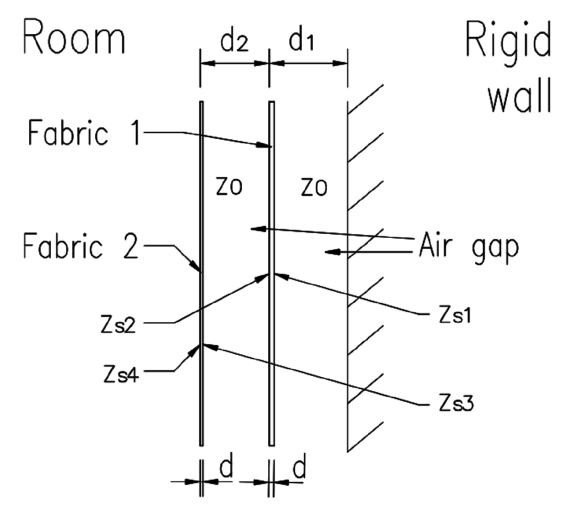

Fig. 1. A schematic sketch explaining construction of the proposed double-layer acoustic curtain.

tem shown in Fig. 1, we will use the formula for the plane wave reflected from a wall with known surface impedance $Z$. For normal incidence of a harmonic wave characterized with wavenumber $k$, the impedance at the distance $d$ from the wall is [10]:

$$
z_{s}(d)=z_{0} \frac{z_{0}-i Z \cot (k d)}{Z-\mathrm{i} z_{0} \cot (k d)},
$$

from which at a distance $d_{1}$ from a perfectly rigid wall $(Z=\infty)$ we obtain

$$
z_{s 1}=-i z_{0} \cot \left(k d_{1}\right) \text {, }
$$

where $z_{0}$ is the characteristic impedance of the medium. The output acoustic impedance for the fabric hanged at a distance $d_{1}$ from a perfectly rigid wall can be expressed as [11]:

$$
z_{s 2}=z_{e 1}+z_{s 1} .
$$

The final step is calculation of the second fabric layer located $d_{2}$ away from the surface with impedance $Z=z_{s 2}$ and characterized wit impedance $z_{e 2}$. Assuming that the angle of incidence of the wave onto the second fabric layer is $\varphi$ and using formula (7) we obtain

$$
z_{s 3}(\phi)=z_{0} \frac{-\mathrm{i} z_{s 2} \cot \left(k d_{2} \cos (\phi)\right)+z_{0}}{z_{s 2}-\mathrm{i} z_{0} \cot \left(k d_{2} \cos (\phi)\right)} .
$$

The resultant impedance of the system can be symbolically expressed as (cf. Eq. (9))

$$
z_{s 4}(\phi)=z_{e 2}+\frac{z_{s 3}}{\cos \phi},
$$

where function $\cos (\phi)$ appearing in denominator of the second term describes dependence on the angle of wave incidence onto the second fabric layer [7].

Given acoustic impedance $z_{s 4}$ of the whole doublelayered structure, its is now possible to determine its sound reflection coefficient [7]

$$
R=\frac{\frac{z_{s 4}}{\rho c} \cos (\phi)-1}{\frac{z_{s 4}}{\rho c} \cos (\phi)+1},
$$

and further, its sound absorption coefficient

$$
\alpha(\phi)=1-|R|^{2}
$$

that, averaged over the whole half-space, can be considered as the sound wave absorption coefficient uniformly distributed incidence [11]:

$$
\alpha_{\text {diff }}=2 \int_{0}^{\pi / 2} \alpha(\phi) \sin 2(\phi) \mathrm{d} \phi .
$$

\section{Experimental and model studies}

\subsection{Verification of the theoretical model}

Taking into account different fabric types and structures of the double-layer curtain, the above-presented analytical model was subjected to experimental verification. Eight different materials were tested with resistance $r=\sigma d$ in the range $400-2752 \mathrm{~Pa} \mathrm{~s} / \mathrm{m}$ and the surface density $0.20-0.35 \mathrm{~kg} / \mathrm{m}^{2}$, at distances from the hard partition varying in the range $0.01-0.30 \mathrm{~m}$ and the distance between individual layers of fabric ranging from $0.01 \mathrm{~m}$ to $0.20 \mathrm{~m}$.

Measurements of the normal incidence sound absorption coefficient were carried out on samples with diameter of $0.1 \mathrm{~m}$ with the use of two-microphone impedance tube according to the standard PN-EN ISO 10534-2:1998 [12].

It has been proven that for the analyzed frequency range $100-1600 \mathrm{~Hz}$, the theoretical model developed for 
double-layered curtains correctly reproduces the sound absorption values obtained from the measurements. Some discrepancies observed in the corresponding graphs as for the resonance frequencies may be a result of the fact that fabric thickness was neglected in the adopted model (Fig. 2).

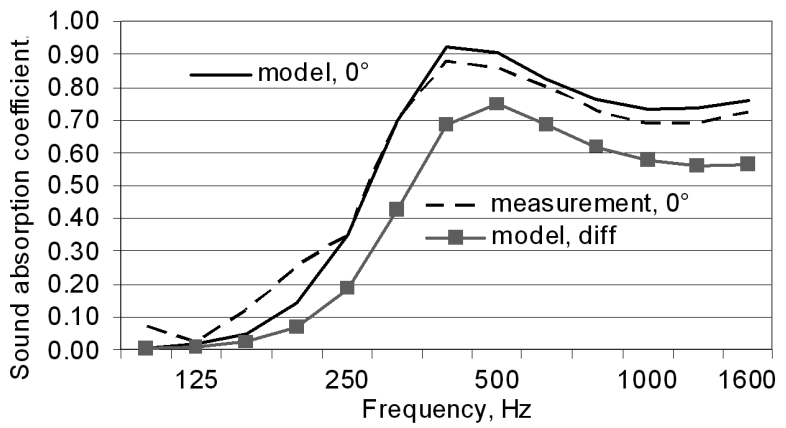

Fig. 2. Frequency characteristics of the sound absorption coefficient for a curtain sample $\left(r_{1}=1234 \mathrm{~Pa} \mathrm{~s} / \mathrm{m}\right.$, $\left.r_{2}=779 \mathrm{~Pa} \mathrm{~s} / \mathrm{m}, d_{1}=0.04 \mathrm{~m}, d_{2}=0.02 \mathrm{~m}\right)$, measured and calculated from the model.

\subsection{Application of two-layer curtains to reduce the reverberation time in concert halls}

The verified model of two-layer acoustic curtains has been used in design work on the fabric structure intended for the main concert hall of the Krakow Philharmonic. The hall demands acoustic adjustment especially in situations when rehearsals are conducted without audience. The reverberation time values in the $2-4 \mathrm{kHz}$ frequency band decrease by more than $5 \%$ and the change is easily perceptible for performers [13]. Among objective parameters that convey such subjective impression of the change, the most appropriate is the early decay time (EDT) that was used for this purpose in [1].

On the grounds of model studies implemented in CATT-Acoustic application it has been determined that to compensate the effect consisting in a change of acoustic absorption of armchairs after taking them by listeners [14], $60 \mathrm{~m}^{2}$ of the acoustic material should be used. As the most favorable configuration, symmetrical placement of the curtains along side walls at the height of auditorium in two strips $3-\mathrm{m}$ high and $10-\mathrm{m}$ long was adopted.

The fabric structure distributed this way allowed to improve the reverberation time characteristics not only on the stage but also in the auditorium area. In view of the necessity to maintain the optimum reverberation time on the stage, the use of a fabric structure for covering the stage walls would be more effective; however, this would also result in reduction of first reflections reaching the musicians and thus in deterioration of both self- and cross-hearing among orchestra members.

From among the analyzed materials, the best results have been obtained for a double-layer structure made of fabric with density $0.35 \mathrm{~kg} / \mathrm{m}^{2}$ and airflow resistivity $2752 \mathrm{~Pa} \mathrm{~s} / \mathrm{m}$ located at a distance 0.1 from the wall and combined with a fabric with surface density $0.25 \mathrm{~kg} / \mathrm{m}^{2}$
TABLE

Uniformly distributed incidence sound absorption coefficient values for curtains used in acoustic model of Krakow Philharmonic and Podkarpacka Philharmonic concert halls.

\begin{tabular}{l|l}
\hline Sound absorption coefficient & Frequency band center, $\mathrm{Hz}$
\end{tabular} values of curtains designed

for concert hall at:

\begin{tabular}{l|l|l|l|l|l|l|l|}
\hline Krakow Philharmonic & 0.07 & 0.71 & 0.41 & 0.31 & 0.53 & 0.70
\end{tabular}

\begin{tabular}{ll|l|l|l|l|l|l|l}
\hline Podkarpacka Philharmonic & 0.12 & 0.57 & 0.50 & 0.51 & 0.39 & 0.38
\end{tabular}

and airflow resistivity $779 \mathrm{~Pa} \mathrm{~s} / \mathrm{m}$ separated from the first one by a $0.02 \mathrm{~m}$ gap. The reverberation sound absorption coefficient of such structure is presented in Table, and the characteristic of the early decay time obtained from modeling the Krakow Philharmonic concert hall before and after introduction of the curtains is shown in Fig. 3.

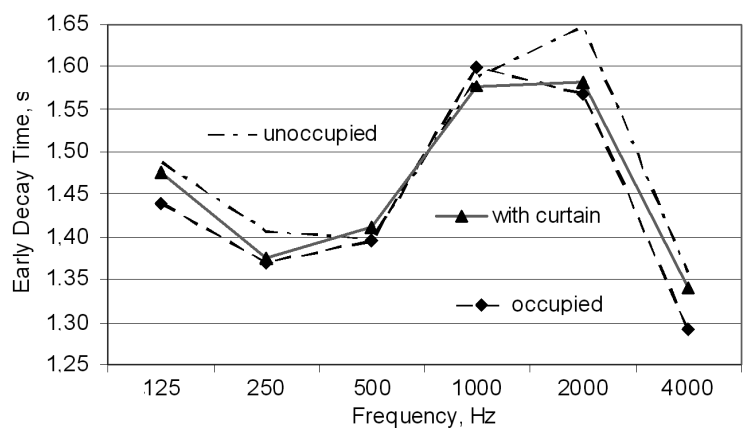

Fig. 3. The early decay time (EDT) characteristics of the Krakow Philharmonic concert hall simulated for different occupation situations.

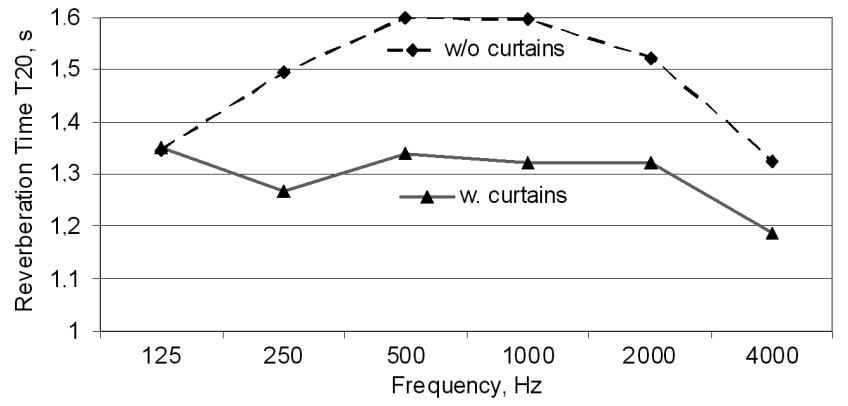

Fig. 4. Reverberation time characteristics of occupied Podkarpacka Philharmonic concert hall simulated for conditions before and after introduction of acoustic curtains.

The rationale behind the proposal to introduce acoustic curtains in the Podkarpacka Philharmonic was the necessity to adapt acoustics of the interior to requirements related to the use of electroacoustic sound amplification system. Acoustics of the interior was originally designed with natural sources of sound being given the priority, while the artificial sound amplification (especially with 
the speech signal) requires much shorter reverberation times. It has been therefore proposed that the backcloth, used typically for theatre performances, will ensure acoustic absorptivity resulting in reduction of the reverberation time value from $T_{m}=1.55 \mathrm{~s}$ to $T_{m}=1.30 \mathrm{~s}$ (Fig. 4). The required effect has been achieved in the acoustic model by using the fabric with sound absorption characteristic presented in Table I.

The acoustic structure of $147 \mathrm{~m}^{2}$ was designed in the form of two layers of a fabric with airflow resistivity $2752 \mathrm{~Pa} \mathrm{~s} / \mathrm{m}$ separated by a gap of $0.09 \mathrm{~m}$ and hanging at a distance of $0.11 \mathrm{~m}$ from the hard wall surface.

\section{Conclusions}

In the framework of the study described in this paper it has been demonstrated that by using two-layer fabric curtains it is possible to control acoustic absorptivity in a wide range and shape this way fundamental acoustic parameters of interiors. A significant improvement of acoustic conditions was obtained for both of the analyzed auditoriums. In the Krakow Philharmonic concert hall, reverberation time differences related to empty and occupied hall have been leveled. Based on numerical simulation, side walls of the auditorium were found to be the best for acoustic treatment in that case. Doublelayer curtain proved also to be a very efficient way to improve acoustic parameters of rooms used for different functions. In the Podkarpacka Philharmonic, a more balanced RT frequency characteristics good for sound amplification systems was obtained. Taking into account results of numerical simulation as well as the functional program of the room, the curtain was used as a backcloth.

\section{Acknowledgments}

This paper was written with the support of the Faculty Dean's Grant No. 15.11.130.210.

\section{References}

[1] L. Beranek, Concert halls and opera houses, Springer, New York 2002.

[2] M. Long, Architectural acoustics, Elsevier, London 2006.

[3] T. Kamisiński, J. Rubacha, A. Pilch, Acta Physica Polonica A 118, 83, (2010).

[4] P. Soltani, M. Zerrebini, Textile Research Journal 82, 875, (2012).

[5] Y.I. Hanna, M.M. Kandil, Applied Acoustics 34, 281, (1991).

[6] ISO 9053, Acoustics - Materials for acoustical applications - Determination of airflow resistance.

[7] H. Kutruff, Room acoustic, Spon Press, London and New York 2009.

[8] K.U. Ingard, Notes on sound absorption technology, Noise Control Foundation, 1994.

[9] T. Wu, T.J. Cox, Y.W. Lam, J. Acoust. Soc. Am. 108, 643 (2000).

[10] T.J. Cox, P. D'Antonio, Acoustic Absorbers and Diffusers, Theory, design and application, Taylor \& Francis, Abingdon 2009.

[11] T. Kamisiński, K. Brawata, A. Pilch, J. Rubacha, M. Zastawnik, Archives of Acoustics 37, 317 (2012).

[12] ISO 10534-2:1998, Acoustics - Determination of sound absorption coefficient and impedance in impedance tubes - Part 2: Transfer-function method.

[13] ISO 3382-1:2009, Acoustics - Measurement of room acoustic parameters - Part 1: Performance spaces.

[14] J. Rubacha, A. Pilch, M. Zastawnik, Archives of Acoustics 37, 483 (2012). 